\title{
Laboratory and synchrotron rocking curve imaging for crystal lattice misorientation mapping
}

\author{
Petr Mikulík, Ondřej Caha, Mojmír Meduňa
}

Masaryk University, Brno, Czech Republic;

mikulik@physics.muni.cz

Recently the rocking curve imaging (RCI) technique has been transferred from synchrotron to laboratory set-ups. RCI is an X-ray diffraction technique which combines full-field X-ray digital topography and Bragg-diffraction rocking curve recording. A large almost parallel monochromatic beam irradiates a crystalline sample with a misorientation distribution characterized by local tilt angles. Series of digital topographs are measured by a two-dimensional detector at different sample orientations from which peak characteristics of millions of local Bragg peaks from each series are extracted. The field of view and lateral resolution is given by the camera size, its pixel size and the Bragg angle, while the angular resolution is given by the rocking curve width being typically much smaller than the misorientation angles of the studied crystal. Simultaneous high spatial resolution provided by the two-dimensional detector and high angular resolution $\left(0.001^{\circ}\right)$ allows to quantify crystalline structure perfectness over large sample area which scales with the area of the detector. Therefore the rocking curve imaging is an imaging method with faster recording compared to usual scanning area diffractometry which requests measurement of the rocking curve at each surface point.

Synchrotron RCI [1,2] profits from large parallel beam, high flux and small detector pixel size downto one micrometre. For small misorientations, detector can have any distance from the sample, while larger misorientations due to inherent focusing/defocusing of the diffracted (micro)beams require a dedicated reconstruction procedure.

Laboratory RCI [3] with a slightly divering beam requires small misorientation angles and very small sample to detector distance, thus a home-made extension for a commercial diffractometer is necessarily. Current two-dimensional detectors available at laboratory diffractometers have typical spatial resolution downto $0.1 \mathrm{~mm}$ which make it possible to analyze a large sample area at once. On several examples, we will show suitability of the method for a characterisation of several large-area semiconductor wafers. In particular, we demonstrate application of the method on mapping of local lattice tilt distortion of large array of SiGe microcrystals epitaxially grown on silicon.

[1] P. Mikulík, et al. (2003) Journal of Physics D: Appl. Phys. 36, A74-A78.

[2] D. Lübbert, et al. (2005) Journal of Applied Crystallography 38, 91-96.

[2] M. Meduňa, et al. (2021) Journal of Applied Crystallography 54.

Keywords: rocking curve imaging, diffraction, misorientation, mapping, crystallites 